\title{
Is a 4-Week Long Functional Training Program Able to Improve Physical Fitness Components in University Faculty and Staff?
}

Original Research

\author{
Rachel Dykstra ${ }^{1}$, Alec Thomson ${ }^{2}$, \& Nicholas Hanson ${ }^{3}$ \\ ${ }^{1}$ Nebraska Wesleyan University, Lincoln, NE/USA \\ ${ }^{2}$ Grand Valley State University, Allendale, MI/USA \\ ${ }^{3}$ Western Michigan University, Kalamazoo, MI/USA
}

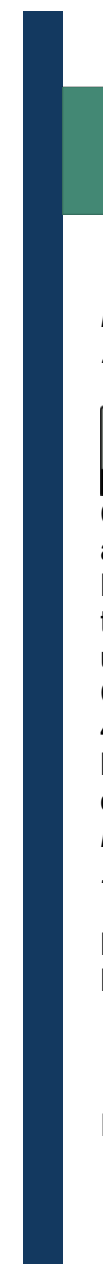

Published: August 9, 2021

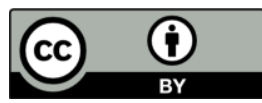

Copyright, 2021 by the authors. Published by Research Directs and the work is licensed under the Creative Commons Attribution 4.0 International License. To view a copy of this license, visit http://creativecommons .org/licenses/by/4.0/

Research Directs in Health Sciences: 2021, Volume 1 (Issue 1): 7

ISSN: 2768-492X
Open Access

\begin{abstract}
Introduction: The physical wellbeing of university faculty and staff is often overlooked. Exercise improves physical fitness and could indirectly lead to benefits such as enhanced work productivity and mood. Therefore, the purpose of this study was to determine the effects of a short duration high-intensity functional training (HIFT) program on essential components of physical fitness in university faculty and staff members.

Methods: Eighteen university faculty and staff members (age $35.4 \pm 11.7$ years) participated in the study. Prior to training, they completed five assessments: cardiorespiratory fitness (submaximal $\mathrm{VO}_{2}$ ), muscular strength (one repetition maximal leg press [1RM]), muscular endurance (push-ups), body composition (body fat percentage $[\mathrm{BF} \%]$ ), and trunk flexibility (sit and reach). Tests were repeated after four weeks (three sessions per week) of multi-modal HIFT. Paired samples t-tests were used to compare pre- and post-training data.

Results: There were significant improvements in cardiorespiratory fitness $(p=0.009)$, $1 \mathrm{RM}$ leg press $(\mathrm{p}<0.01)$ and push-ups completed $(\mathrm{p}<0.01)$. No significant changes were shown in $B F \%(p=0.581)$ or trunk flexibility $(p=0.199)$.

Conclusions: Four weeks of training improved cardiorespiratory fitness as well as muscular strength and endurance. HIFT programs represent a time-efficient and effective training method for improving multiple components of physical fitness in university faculty and staff, despite a relatively short duration.
\end{abstract}

Key Words: multi-modal training, cardiorespiratory fitness, high intensity exercise

Corresponding author: Rachel Dykstra, rdykstr2@nebrwesleyan.edu

\section{Introduction}

Cardiorespiratory fitness (CRF), muscular strength and endurance, body composition, and flexibility are considered the five most important components of physical fitness ${ }^{1}$ and improvements in these areas have been associated with maximizing health benefits ${ }^{1}$. High-intensity interval training (HIIT) has been consistently shown to augment these physical fitness components, and improvements have occurred after as little as a 3-week training period ${ }^{2}$. Increases in $\mathrm{VO}_{2} \mathrm{max}$ (a measure of $\mathrm{CRF}$ ) are most commonly reported ${ }^{2,3}$, as HIIT originally implemented a limited number of modalities (i.e., running, cycling, and/or rowing). However, previous research suggested that progressions in muscular strength and endurance could be achieved through HIIT if additional modalities are incorporated into the workout regimen ${ }^{4}$. Therefore, multi-modal HIIT has surfaced, often referred to 
as high-intensity functional training (HIFT), which uses a combination of aerobic and strength training with aims to improve all components of physical fitness ${ }^{5}$.

With promising positive physiological results emerging from HIFT ${ }^{6}$, many companies have created workout regimens that utilize variations of this format, encouraging individuals to join a "community" that emphasizes not only fitness, but overall health and wellness. It has been shown that the overall impact of these training communities (e.g., CrossFit) can elicit high levels of sense of community, satisfaction, and motivation ${ }^{7}$. One such HIFT community, Functional 45 (F45), emerged in 2011 and integrates team-based functional-HIIT and circuit-based workouts within a 45-minutes session. The F45 database holds over 4,000 exercises and 36 exclusively formatted classes, which means that each $45-$ minute class is unique and will never be repeated throughout participation ${ }^{8}$. The functional training method used recreates every day functional movements, which does not only target improving physical fitness, but feeling confident with day-to-day activities ${ }^{8}$. Moreover, this training can be modified for an array of fitness levels and has the potential to improve a broad range of physical fitness components while remaining time-efficient 7 . By decreasing the time required, but still providing an effective workout, HIFT is appealing to a variety of individuals, one such being university employees.

Research on health-related interventions in university faculty and staff is limited, as the majority of collegiate campuses have a strong focus on student-based health intervention programs ${ }^{9}$. It is important to consider the health and wellness of university faculty and staff, as this population faces the same environment as students, while being on campus for extended and sometimes inflexible hours ${ }^{10}$. Developing a positive work environment that balances the fostering of health and optimal work performance is critical in order to combat negative physical and psychological health risks ${ }^{10}$. Preceding research on faculty and staff-based health interventions has emphasized that these programs are influential for decreases in absences, improvement in productivity, and influence significant gains in financial and physical health 9, 11, 12. Therefore, the purpose of this study was to examine the effects of a four-week HIFT program on the five essential components of physical fitness: cardiorespiratory fitness, muscular strength and endurance, body composition, and flexibility, in university faculty and staff. We hypothesized that the HIFT program would significantly improve $\mathrm{VO}_{2} \max$ and muscular strength and endurance, as HIFT emphasizes improving both aerobic and anaerobic capacity.

\section{Scientific Methods}

\section{Participants}

All procedures performed in studies involving human participants were in accordance with the ethical standards of the institutional committee and with the 1964 Helsinki Declaration and its later amendments or comparable ethical standards. This research study was approved by the Human Subjects Institutional Review Board and all subjects were informed of the benefits and risks of investigation prior to signing the informed consent document. The study included 18 individuals ( 6 males, 12 females; (age $35.4 \pm 11$ years) who were faculty and staff members of a midwestern university and were also members of the F45 program on campus. An American College of Sports Medicine (ACSM) health screening document was completed by each participant, prior to participating in the study, in order to ensure a low-risk status (1 or less cardiovascular disease risk factors). A Physical Activity Readiness Questionnaire (PAR-Q+) was also completed in order to screen individuals for symptoms or medical conditions that would contraindicate exercise. Individuals who experienced any musculoskeletal injury within the last 6 months were unable to participate.

\section{Protocol}

Pre-Training

Each subject reported to the laboratory for two visits (pre- and post-training). Height and weight were measured and recorded and then subjects were familiarized with laboratory equipment and the experimental protocols. Prior to completing any tests, the rate of perceived exertion (RPE) scale ${ }^{13}$ was described in detail and subjects were asked to practice exercises at each specified RPE to ensure understanding of the scale. The order of testing followed the guidelines of ACSM 1 .

Body Composition

Body composition was assessed using the In-Body 570 body composition scale (Hangzhou, China). Subjects were asked to stand barefoot on the scale and follow the prompts (i.e., holding or not holding the handles) while the scale measured their body composition. Total body fat percentage $(\mathrm{BF} \%)$ was recorded. 
Cardiorespiratory Fitness

Subjects were fitted with a chest strap heart rate monitor (Polar T34; Kempele, Finland) and instructed to complete a five-minute warmup on a cycle ergometer (Wattbike Pro, Woodway; Nottingham, United Kingdom) at a self-selected intensity. The submaximal protocol was a modified version of the self-paced $\mathrm{VO}_{2} \max (\mathrm{SPV})$ test ${ }^{14}$. The test was exactly eight minutes in length, separated into four, two-minute stages, perceptually regulated using RPE values of 11 (light), 13 (somewhat hard), 15 (hard), and 17 (very hard), in that order. Subjects were allowed to adjust their cadence and resistance for the duration of the test in order to maintain the prescribed RPE for each state. Upon completion of the test, subjects cooled down at a self-selected pace for a minimum of three minutes.

A TrueOne 2400 metabolic cart (ParvoMedics, Sandy, UT, United States) was used to collect all expired gases during the submaximal $\mathrm{VO}_{2} \max$ test. Flow and gas calibrations were performed 5-10 minutes prior to each test to ensure accurate recording. Fifteen-breath rolling averages were used to determine maximal values for ventilation $\left(\mathrm{V}_{\mathrm{E}}\right)$, carbon dioxide production $\left(\mathrm{VCO}_{2}\right)$, relative oxygen consumption $\left(\mathrm{VO}_{2}\right)$, respiratory exchange ratio (RER), and heart rate (HR).

Because a submaximal test was used, it was necessary to determine the estimated $\mathrm{VO}_{2}$ max which would theoretically occur at RPE20. First, predicted maximal heart rate (HRmax) was determined using the Tanaka formula (i.e., 208 $0.7 \mathrm{x}$ age) ${ }^{15}, 16$. For $\mathrm{HR}$ and $\mathrm{VO}_{2}$, the last 15 seconds of each stage of the SPV were averaged. These values were plotted against each other for each stage; a best fit line was then used to obtain a regression equation and determine the slope of the line. The constant from the regression equation (b), each subject's HRmax (x) and the slope (m) were input into the linear regression equation to solve for predicted $\mathrm{VO}_{2} \mathrm{max}$.

\section{Muscular Strength}

Following the test on the cycle ergometer, subjects were given adequate time to rest (approximately ten minutes) before implementing a 1-repetition maximum (1RM) leg press. Subjects warmed up by completing ten repetitions with submaximal weight. Thereafter, an initial weight was chosen that was within $50-70 \%$ of the subject's perceived maximal capacity. If the resistance was too light, it was progressively increased by $2.5-20 \mathrm{~kg}$ until the subject could no longer complete multiple repetitions and was only able to implement one repetition with proper form and range of motion. Two researchers were present during each maximal test to spot subjects and ensure proper form was being implemented. Once this test was complete, the subjects were given approximately ten minutes to rest before the muscular endurance test started.

\section{Muscular Endurance}

To test for muscular endurance, a simple push-up test was implemented; the protocol differed between sexes. Male subjects were instructed to start in a down position (hands pointing forward and under shoulders, back straight, head up, using the toes as a pivotal point), and females were instructed to start in the modified "knee push-up" position (legs together, lower leg in contact with mat, ankles plantar flexed, back straight, hands shoulder width apart, head up, using knees as the pivotal point). All subjects were instructed to raise their body until their elbows were completely straight, and then return to the down position, where their chin touched the mat. Subjects were then instructed to complete their maximum number of consecutive push-ups, without rest.

\section{Flexibility}

The Canadian Trunk Forward Flexion test was used to assess flexibility. Subjects were instructed to sit on the ground, without shoes, and with the soles of their feet flat against the flexometer (sit-and-reach box) at the $26 \mathrm{~cm}$ mark. They then slowly reached forward with body hands, as far as possible, holding this position for two seconds. Subjects' hands were to be kept parallel and overlapped when reaching forward. The research ensured that the legs of the participant were kept straight and instructed subjects to continue breathing normally throughout the test. Three trials of this test were completed, and the maximal value was recorded.

\section{F45 Training}

After pre-training was complete, the subjects were instructed to attend F45 classes a minimum of three times per week for four consecutive weeks. Classes were conducted in a certified F45 room at the university recreation center. Each class was 45 minutes in length and the order and type of exercises implemented were unique to each class, which helped target various muscular groups between and within sessions. Classes were instructed by certified F45 coaches who used HIFT, which incorporates both resistance and aerobic based exercises. Subjects were asked to record all classes they attended for verification of attendance. The average number of classes that were attended within the four 
weeks of training was $14.3 \pm$ 3.6. All subjects had less than one year of experience participating in F45 classes. Testing after the training period included a second visit to the laboratory, where all pre-training tests were repeated.

Statistical Analysis

All data was analyzed using IBM SPSS Statistics (Version 25, Armonk, NY). Paired-samples t-tests were performed to compare dependent variables (mass, $\mathrm{BF} \%$, predicted $\mathrm{VO}_{2} \mathrm{max}, 1 \mathrm{RM}$ leg press, push-up repetitions, flexibility) pre- and post-training. 95\% confidence intervals (CIs) were also calculated. Significance level was set $a$ priori at $<.05$. Cohen's $d$ values were calculated to show the effect size.

\section{Results}

Pre- and post-training results are shown in Table 1.

Table 1. Physical Fitness Values Pre- and Post-F45 Training.

\begin{tabular}{lcccc}
\hline Variables & Pre & Post & $\boldsymbol{p}$ & $\boldsymbol{d}$ \\
Body Fat (\%) & $26.7 \pm 8.3$ & $26.5 \pm 7.8$ & 0.581 & .03 \\
VO $_{2}$ max $\left(\mathbf{m l} \cdot \mathbf{k g}^{-1} \cdot \mathbf{m i n}^{-1}\right)$ & $38.2 \pm 8.1$ & $39.8 \pm 7.0$ & $.009 *$ & .22 \\
Leg Press 1RM (kg) & $174.5 \pm 73.6$ & $197.7 \pm 75.4$ & $<.001 *$ & .31 \\
Push-ups (repetitions) & $35.7 \pm 9.5$ & $42.7 \pm 13.8$ & $<.001 *$ & .59 \\
Sit-and-reach (cm) & $33.7 \pm 7.0$ & $34.4 \pm 6.4$ & .199 & .12 \\
\hline
\end{tabular}

Note. Data is presented as mean \pm SD. $*$ denotes a significant difference between pre- and post-training. Effect size given as Cohen's $d$ (small: 0.2 , medium: 0.5 , large: 0.8 ).

\section{Body Composition}

No significant differences were found for body fat percentage ( $\mathrm{BF} \%)$ between pre- and post-training $(\mathrm{p}=.581, d=.03)$. The pre-training BF\% was $26.7 \pm 8.3 \%$ and was $26.5 \pm 7.8 \%$ post-training. This computed to a percentage change of $-0.75 \%$.

\section{Cardiorespiratory Fitness}

There was a significant difference between predicted $\mathrm{VO}_{2} \max$ pre- and post-training $(\mathrm{p}=.009, d=0.22$ ). Prior to training, the average $\mathrm{VO}_{2} \max$ was $38.2 \pm 8.1 \mathrm{ml} \cdot \mathrm{kg}^{-1} \cdot \mathrm{min}^{-1}$, increasing to $39.8 \pm 7.0 \mathrm{ml} \cdot \mathrm{kg}^{-1} \cdot \mathrm{min}^{-1}$ post-training. The mean improvement was $1.6 \mathrm{ml} \cdot \mathrm{kg}^{-1} \cdot \mathrm{min}^{-1}(95 \%$ CI $[0.5,2.8])$. A percentage change of $4.19 \%$ was found. Individual differences between pre- and post-training are shown in Figure 1.

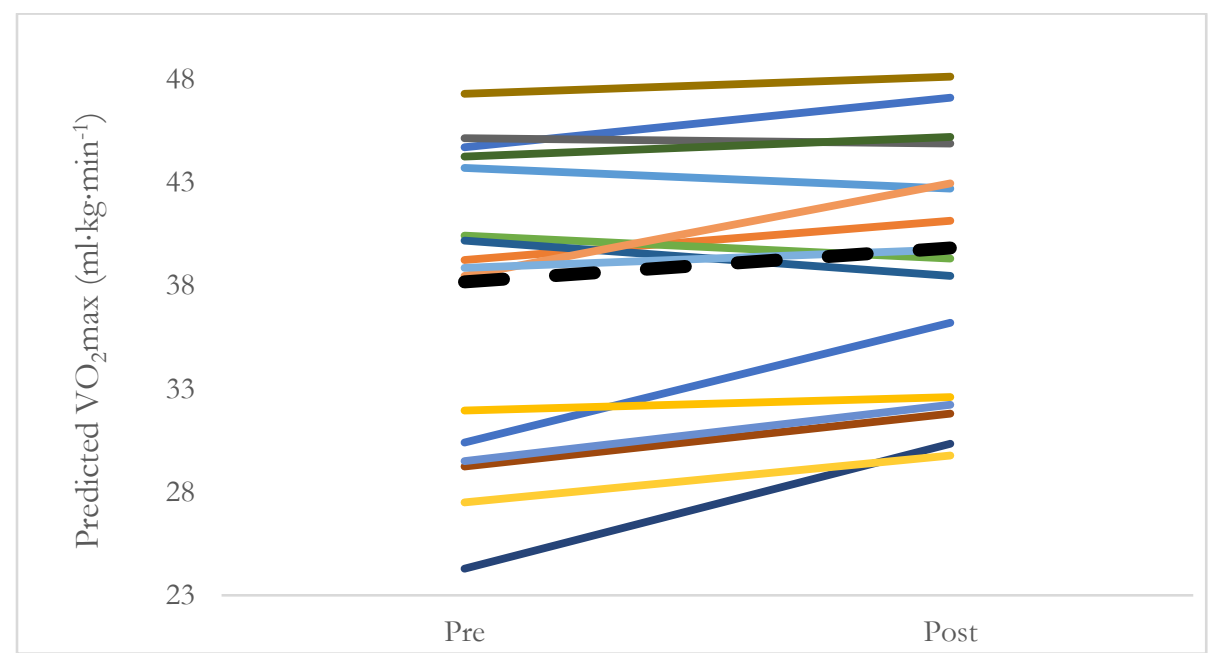

Figure 1. Change in predicted maximal oxygen consumption $\left(\mathrm{VO}_{2} \mathrm{max}\right)$ for all subjects. Dotted line represents the mean change.

Muscular Strength

There was a significant improvement in muscular strength after training $(\mathrm{p}<.001, d=0.31)$. Average values pre-training for $1 \mathrm{RM} \mathrm{leg}$ press were $174.5 \pm 73.6 \mathrm{~kg}$ and increased to $197.7 \pm 75.4 \mathrm{~kg}$ post-training, totaling to a percentage change of $13.30 \%$, and a mean improvement of $51.1 \mathrm{~kg}(95 \%$ CI $[35.8,66.5])$. Individual pre- and post-training differences in 1RM leg press are presented in Figure 2. 


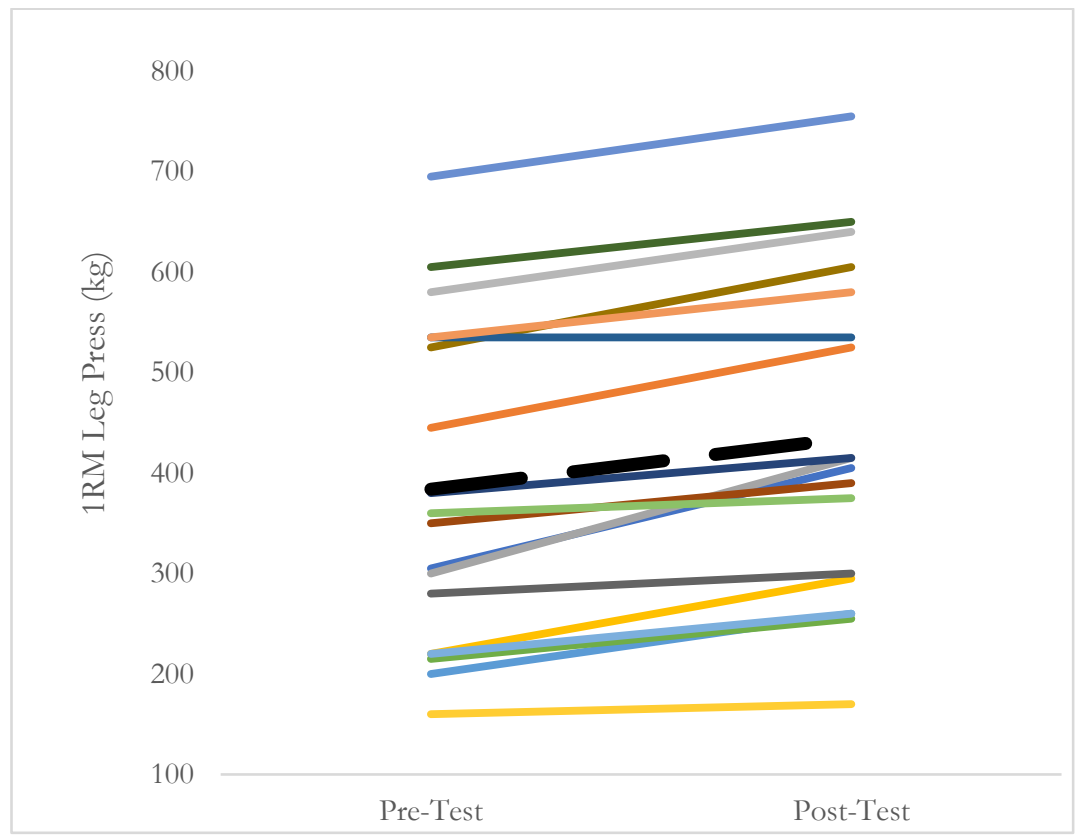

Figure 2. Change in one-repetition maximum (1RM) for all subjects. Dotted line represents the mean change.

\section{Muscular Endurance}

A significant difference was shown between the pre- and post-training push-up test $(\mathrm{p}<.001, d=0.59)$. An average of $35.7 \pm 9.5$ repetitions were completed prior to training, while $42.7 \pm 13.8$ were completed post-training (Figure 3). There was a percentage change of $19.61 \%$. The mean improvement for this variable was 7 repetitions (95\% CI [3.6, 10.4]).

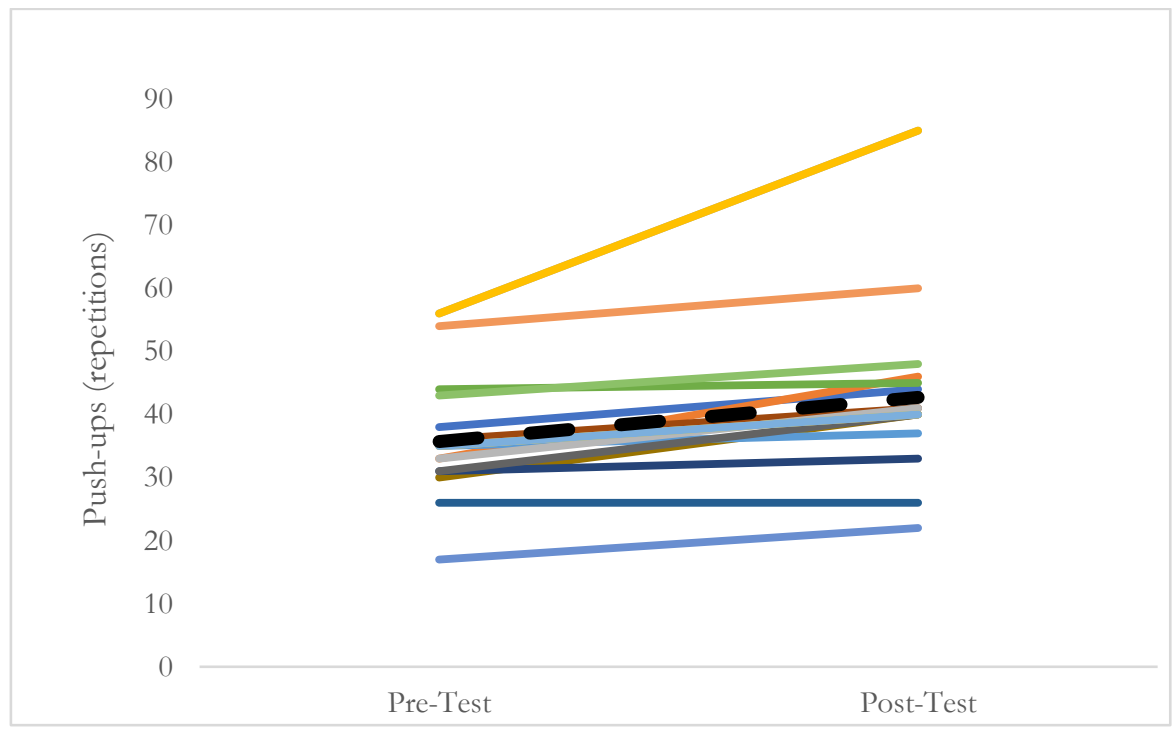

Figure 3. Change in push-up repetitions for all subjects. Dotted line represents the mean change. 
Flexibility

There was no difference in flexibility (pre-training: $33.7 \pm 7.0 \mathrm{~cm}$; post-training: $34.4 \pm 6.4 \mathrm{~cm}$; percentage change: $2.08 \%, p=.199, d=0.12)$.

\section{Discussion}

The main purpose of this study was to determine the effects of a 4-week, F45 HIFT program on the five major components of physical fitness, using university faculty and staff members as subjects. There were significant improvements in CRF, muscular strength, and muscular endurance, confirming our hypothesis. No changes occurred in flexibility or body composition. To our knowledge, this is the first time that physical fitness components of university faculty and staff have been assessed before and after a relatively short duration of HIFT. Our hypothesis was supported, and the results suggest that F45 training for only four weeks can lead to marked improvements in multiple areas of physical fitness, further supporting HIFT as an effective method of exercise.

Aerobics Responses to HIFT

The relationship between CRF and health risks is well established, as CRF is a strong predictor of cardiovascular disease and all-cause mortality ${ }^{1}$. Due to this, a common goal in exercise prescriptions is to prioritize physical activity and exercise programs that augment CRF, further preventing these diseases and maintaining an optimal level of health. Numerous studies implementing HIFT protocols have shown the ability for this type of training to improve CRF $>$ Menz et al. ${ }^{17}$ conducted a four-week functional training program with 18 regularly active participants; $\mathrm{VO}_{2} \mathrm{max}$ significantly improved post-training (pre: $49.5 \pm 6.6 \mathrm{ml} \cdot \mathrm{kg}^{-1} \cdot \mathrm{min}^{-1}$, post: $54.5 \pm 5.3 \mathrm{ml} \cdot \mathrm{kg}^{-1} \cdot \mathrm{min}^{-1} ; \mathrm{p}<.01$ ). Another study ${ }^{18}$ explored the effects of a short-duration HIFT program on muscular strength and aerobic/anaerobic capacity. Twenty recreationally active individuals (age $26.6 \pm 1.9$ years), with no prior experience with HIFT programs, were recruited for the study. All participants trained at a local gym for a minimum of three days/week for four weeks. There were significant improvements in $\mathrm{CRF}<$ anaerobic capacity, and muscular strength (all $\mathrm{p}<.05)$. Our study also showed improvements in CRF after only four weeks of training, corroborating these previous findings. Further research is suggested to account for mechanisms behind early aerobic adaptations ${ }^{7,19}$ during HIFT and whether these changes will continue throughout longer lengths of training or when coupled with other training methods.

\section{Muscular Responses to HIFT}

Because HIFT utilizes both the aerobic and anaerobic energy systems during a single session, this type of concurrent training is appealing for many populations ${ }^{5}$ and has been shown to elicit muscular adaptations and physiological responses (e.g., muscular hypertrophy, enhanced anaerobic power, and improved agility ${ }^{7}$ ), which is noteworthy for athletes. Crawford et al. ${ }^{19}$ used a HIFT intervention in an attempt to improve physiological measures of fitness (CRF, muscular endurance, muscular strength) in recreationally active individuals (ages $22.6 \pm 3.5$ years). Within an eightweek time frame, there were no differences in pre- and post-CRF, but there were significant improvements in maximal strength for a squat $(\mathrm{p}<.01)$ and deadlift $(\mathrm{p}<.01)$. Another study ${ }^{4}$ examined the effects of a multimodal, circuit HIFT on improvements in muscular strength for a $1 \mathrm{RM}$ with back squat, shoulder press, and deadlifts and muscular endurance with repetitive back squats to exhaustion. There were significant increases in squat, shoulder press, and deadlift strength. Similarly, Myers et al. ${ }^{20}$ concluded that muscular strength for the vertical chest press and hamstring curl both improved throughout HIFT, eliciting results similar to a traditional resistance training combined with aerobic exercise. This represents the ability of HIFT to be a time-efficient and effective method for improving health determinants ${ }^{20}$. Comparably, our study found increases in muscular strength, specifically with the 1RM leg press. Improvements in muscular strength suggest that HIFT can influence increases in anaerobic performance measures through metabolic adaptations ${ }^{4}$. It is quite possible that each of the HIFT programs previously mentioned incorporated exercises that targeted each of the improved muscle groups directly. Because F45 incorporates a wide variety of exercises, further research would need to document the exact exercises implemented during each session individuals partake in, in order to analyze whether or not there is a direct correlation between exercises implemented and increased strength in specific muscle groups.

Body Composition Responses to HIFT

It is likely that improvements in body composition were not present in the current study due to the relatively short duration of the training program. In previous research showing improvements in body composition, HIFT programs lasted at least six weeks ${ }^{21,22}$, whereas the current study was only four weeks in duration. Nevertheless, the current study did show a trend towards improving body composition, but the change was not significant $(\mathrm{p}=.581)$. In the current study, subjects were currently participating in an F45 program prior to participation in the study and had an average $\mathrm{BF} \%$ of $2.67 \pm 8.3 \%$, which is lower than the majority of previous subject groups ${ }^{22,23}$. For example, Wewege et al. ${ }^{22}$ 
had subjects with a beginning $\mathrm{BF} \%$ of $43.9 \pm 5.8 \%$ and subjects in the Brown et al. ${ }^{23}$ study began with $34.5 \pm 6.2 \%$ in a multi-modal group and $36.2 \pm 6.1 \%$ in a rowing group. Subjects in many studies were overweight or obese 22,23 which makes improvements in body composition more feasible, as visceral fat content is initially greater. The potential reason that many studies that do not show a significant loss of BF\% with HIFT is lack of control over the diet of subjects. Even if energy expenditure increases because of participation in physical activity or exercise, dietary consumption levels could still create a calorie excess rather than a deficit ${ }^{24}$. Consequently, changes in body composition are unlikely without the inclusion of caloric restriction or a more adequate caloric intake to produce fat loss and augment muscular gains ${ }^{24}$.

\section{Population Response to HIFT}

Because modality, duration, and type of exercise can all be uniquely chosen for each session, HIFT programs such as F45 can be individualized to mimic the demands of a specific sport or metabolic requirements for a particular population ${ }^{5}$, making this training even more comprehensive for a variety of populations and different fitness abilities (e.g., novice vs. experienced). Heinrich et al. ${ }^{25}$ investigated exercise adherence and enjoyment between different training protocols. Twenty-three inactive participants were randomly assigned to either a HIFT group or a conventional training program; the HIFT group had higher levels of enjoyment and significant improvements in exercise adherence after the eight-week program. All participants in the HIFT group intended to continue the same format of exercise, compared to only $55.6 \%$ of the conventional exercise group. This suggests that HIFT is a time-efficient and safe for the general population, and well-accepted by its participants. HIFT requires individuals to devote less time to exercise in comparison to traditional protocols, which is seemingly beneficial for many populations, such as university employees ${ }^{10}$ who have expressed having limited time for extracurriculars ${ }^{10}$. Additionally, numerous studies have shown that individuals are more likely to participate in exercise programs if they find them to be enjoyable ${ }^{6}$.

Lastly, although perceived benefits from health interventions on university campuses have been presented, still, the focus of most of these programs is on the health and wellness of the students, rather than fostering programs that target both students and faculty and staff ${ }^{9}$. In a recent study ${ }^{12}$, it was noted that the majority $(89.8 \%)$ of faculty and staff had the desire to participate in on-campus exercise programs, with less than $6 \%$ having a concern with student involvement, meaning faculty and staff would most likely be willing to integrate themselves into what was originally "student-based" programs. Because of this, our study is not only aiming to present physical health benefits in university faculty and staff participating in on-campus health programs but is encouraging future researchers to investigate this population further, as positive health modeling by faculty and staff can create a more positive exposure for students 12 .

It is important to note that although most subjects in the current study already had experience with participating in F45 training, improvements in multiple areas of physical fitness were still shown. If subjects with no experience were used, the improvements would likely be greater. Future research should focus on the progress of F45 participants over the span of 8-12 weeks, or even long-term (greater than 1 year) to see the impact this training has on all components of physical fitness for an extended duration. Developing more evidence of the long-term effects of F45 could help further support F45 as a program for maintaining health and promoting the enhancement of components of physical fitness. Moreover, researchers should consider each exercise that each F45 training session executes. This could determine which particular muscular groups are targeted throughout each training and could help develop future hypotheses on whether or not muscular strength gains are possible in various large muscle groups, besides the quadriceps, when implementing this training program.

As with all studies, there were certain limitations present. We implemented a repeated-measures design where subjects served as their own control. Future studies using HIFT interventions may consider including an additional comparison group (e.g., HIIT or traditional resistance training), or a sedentary control group for further interpretation of the variance between exercise interventions. Exercise parameters such as RPE, heart rate, blood lactate, etc., were not assessed during training; however, certified F45 instructors are instructed to ensure that all participants were performing optimally and at the requested intensity levels. Lastly, some, but not all, F45 training sessions were conducted by the researchers; some sessions were instructed by other certified F45 instructors who were not involved with data collection. However, the nature of this study emphasized ecological validity. Moreover, the study portrayed that laboratory monitored training (which is not representative of practical training methods) is not necessary for producing physiological improvements. 


\section{Conclusions}

After completing four weeks of HIFT training, $\mathrm{VO}_{2} \max , 1 \mathrm{RM}$ leg press, and push-up repetitions significantly increased. This indicates improved CRF (and therefore lower risk of all-cause mortality), and gains in muscular strength and endurance, respectively. This training did not, however, improve body composition or flexibility. HIFT represents a time-efficient and effective training method for improving components of physical fitness, despite a short duration (4 weeks in this instance). Moreover, HIFT sessions are quite brief, but effective, creating opportunity of decreasing physical fitness training time without decreasing health benefits and allowing for more time to be devoted to other skills and tasks, which could be highly beneficial for university employees who express time constraints.

\section{Conflicts of Interest}

No conflict of interest.

\section{References}

1. ACSM. American College of Sports Medicine’s Exercise Testing and Prescription. Lippincott Williams \& Wilkins; 2017.

2. Astorino TA, Allen RP, Roberson DW, Jurancich M. Effect of high-intensity interval training on cardiovascular function, VO2max, and muscular force. The Journal of Strength and Conditioning Research. 2012;26(1):138145.

3. Tabata I, Nishimura K, Kouzaki M, et al. Effects of moderate-intensity endurance and high-intensity intermittent training on anaerobic capacity and VO2max. Medicine and Science in Sports Exercise. 1996;28:1327-1330.

4. Buckley S, Knapp K, Lackie A, et al. Multimodal high-intensity interval training increases muscle function and metabolic performance in females. Journal of Applied Physiology, Nutrition, and Metabolism. 2015;40(11):1157-1162.

5. Neto F, Henrique J, Kennedy MD. The Multimodal Nature of High-Intensity Functional Training: Potential Applications to Improve Sport Performance. Journal of Sports. 2019;7(2):33.

6. Feito Y, Heinrich K, Butcher S, Poston W. High-intensity functional training (HIFT): Definition and research implications for improved fitness. Sports. 2018;6(3):76.

7. Claudino JG, Gabbett TJ, Bourgeois F, et al. Crossfit overview: systematic review and meta-analysis. Journal of Sports Medicine. 2018;4(1):11.

8. Functional 45. https:// f45training.com/why-f45-works/

9. Das BM, Rinaldi-Miles AI, Evans EM. Exploring faculty and staff PA barriers at a large university. Journal of Californian Health Promotion. 2013;11(2):61-72.

10. Rebold MJ, Kobak MS, Peroutky K, Glickman EL. The effects of a 12-week faculty and staff exercise program on health-related variables in a university setting. International Journal of Exercise Science. 2015;8(1):49.

11. Haines DJ, Davis L, Rancour P, Robinson M, Neel-Wilson T, Wagner S. A pilot intervention to promote walking and wellness and to improve the health of college faculty and staff. Journal of American College Health. 2007;55(4):219-225.

12. Bright DR, Terrell SL, Rush MJ, et al. Employee attitudes toward participation in a work site-based health and wellness clinic. Journal of Pharmacy Practice. 2012;25(5):530-536.

13. Borg G. Borg's perceived exertion and pain scales. Human Kinetics; 1998.

14. Faulkner J, Mauger AR, Woolley B, Lambrick D. The efficacy of a self-paced VO2max test during motorized treadmill exercise. International Journal of Sport Physiology Performance. 2015;10(1):99-105.

15. Nes B, Janszky I, Wisloff U, Stoylen A, Karlsen T. Age-predicted maximal heart rate in healthy subjects: The HUNT Fitness Study. Scandinavian Journal of Medicine Science in Sports. 2013;23(6):697-704.

16. Tanaka H, Monahan K, Seals D. Age-predicted maximal heart rate revisited. Journal of the American College of Cardiology. 2001;37(1):153-156.

17. Menz V, Marterer N, Amin SB, Faulhaber M, Hansen AB, Lawley JS. Functional Vs. Running Low-Volume High-Intensity Interval Training: Effects on VO2max and Muscular Endurance. Journal of Sports Science \& Medicine. 2019;18(3):497.

18. Kliszczewicz B, McKenzie M, Nickerson B. Physiological adaptations following a four-week of high-intensity functional training. Vojnosanitetski Pregled. 2019;76(3):272-277.

19. Crawford DA, Drake NB, Carper MJ, DeBlauw J, Heinrich KM. Are Changes in Physical Work Capacity Induced by High-Intensity Functional Training Related to Changes in Associated Physiologic Measures? Sports. 2018;6(2):26. 
20. Myers TR, Schneider MG, Schmale MS, Hazell TJ. Whole-body aerobic resistance training circuit improves aerobic fitness and muscle strength in sedentary young females. The Journal of Strength and Conditioning Research. 2015;29(6):1592-1600.

21. Heydari M, Freund J, Boutcher SH. The effect of high-intensity intermittent exercise on body composition of overweight young males. Journal of Obesity. 2012;2012

22. Wewege M, Van Den Berg R, Ward R, Keech A. The effects of high-intensity interval training vs. moderateintensity continuous training on body composition in overweight and obese adults: a systematic review and metaanalysis. Obesity Reviews. 2017;18(6):635-646.

23. Brown EC, Hew-Butler T, Marks CR, Butcher SJ, Choi MD. The Impact of Different High-Intensity Interval Training Protocols on Body Composition and Physical Fitness in Healthy Young Adult Females. Journal of BioResearch. 2018;7(1):177-185.

24. Swift DL, Johannsen NM, Lavie CJ, Earnest CP, Church TS. The role of exercise and physical activity in weight loss and maintenance. Progress in Cardiovascular Diseases. 2014;56(4):441-447.

25. Heinrich K, Patel P, O’Neal J, Heinrich B. High-intensity compared to moderate-intensity training for exercise initiation, enjoyment, adherence, and intentions: an intervention study. BMC Public Health. 2014;14(1):789. 